\title{
A Review Of Reverse Osmosis Membrane Fouling: Formation and Control
}

\author{
Rizka Mulyawan ${ }^{1}$, Agam Muarif ${ }^{2 *}$ \\ ${ }^{1}$ Department of Chemical Engineering, Faculty of Engineering Faculty, Universitas Malikussaleh, Aceh, Indonesia \\ ${ }^{2}$ Department of Material Engineering, Faculty of Engineering Faculty, Universitas Malikussaleh, Aceh, Indonesia \\ *Corresponding authorE-mail: amuarif@ unimal.ac.id
}

Manuscript received 15 April 2021; revised 1 May 2021; accepted 15 June 2021. Date of publication 3 July 2021

\begin{abstract}
Membrane application in reverse osmosis (RO) membrane is getting more attention especially in producing drinking water. However, RO membrane faces challenges that reduces its performance such as its permeation flux, salt rejection, additional energy demand, lifetime decrease, extra pre-treatment process, cleaning and maintenance. The challenge is the formation of fouling. RO membrane fouling can happen inside or outside the membrane and the characteristics of membrane fouling differs from one type to other types, depending on the nature and location of membrane fouling. There are several types of RO fouling, which are Biofouling, Organic Fouling, Inorganic Fouling and Colloidal Fouling. The causes of RO membrane are different from one to another. The properties and materials of the solution entering RO membrane are important as it affects the type of fouling of RO membrane fouling. All of the RO membrane foulings need to be considered during membrane usage and demand solution to be controlled. In order to control the fouling in Reverse Osmosis membrane, there have been several control solutions discovered to the membrane fouling challenges. The control solutions are specified to each one of the fouling, in spite of wide applications for some of it. The control solutions are pre-treatment, which has many methods such as photo oxidation, coagulation, scale inhibitor, ion exchange resins, granular media and membrane treatment, membrane monitoring, membrane cleaning, surface modification, and material addition to membrane or novel membrane material. With various control solutions discovered, the RO membrane still faces fouling issue and is still demanding some more advanced applicable control solutions.
\end{abstract}

Keywords: Fouling, Fouling Control, Membrane Fouling, RO membrane.

\section{Introduction}

In daily life, the application of Chemical engineering is becoming more necessary. Some of the application of Chemical Engineering principle are in food processing industry, pharmaceutical, cosmetics, water purification, and seawater desalination. In those applications, the use of membrane technology takes an important role. Hence, from its applications and potential usages in future, membrane technology has got promising potential especially in the future[1][2][3].

More specifically, the utilisation of membrane in water treatment has been widely applied and is getting more important. On the grounds that fresh and drinkable water demand increases as the population increases. However, solely depending on the ground water does not meet the increasing demand. Consequently, alternative water sources are necessary and have been applied such as water reuse and seawater desalination[4], [5]. On the other hand, there are challenges in utilising both used water and desalination of seawater. The challenges are that the quality of water source is mostly far from the ideal conditions to meet fresh water criteria. Usually, the sources of used water are agricultural residue, sewage, and industrial waste[6]. These challenges make the utilized membrane technology and its sustainability are critical.

One of membrane technology that has been widely used especially in water treatment is reverse osmosis (RO). Osmosis is the process of solvent or water from a solution that has lower solute concentration through a semipermeable membrane to solution that has higher solute concentration[7]. The driving force in osmotic process is concentration difference between the two sides of membrane. Beside reverse osmosis (RO), there are also other processes in membrane technology based on osmotic process. Namely, the processes are forward osmosis (FO) and pressure retarded osmosis (PRO)[8]. In this review, the focus is on fouling on reverse osmosis (RO); its formations strategies to encounter them. The difference is that in reverse osmosis process the flow of water or solvent is in opposite direction to water flow in osmotic process. This is due to external pressure, which is hydrostatic pressure, applied to the higher solute concentration solution[7]. The schematic process of reverse osmosis is shown in Figure 1[9]. 


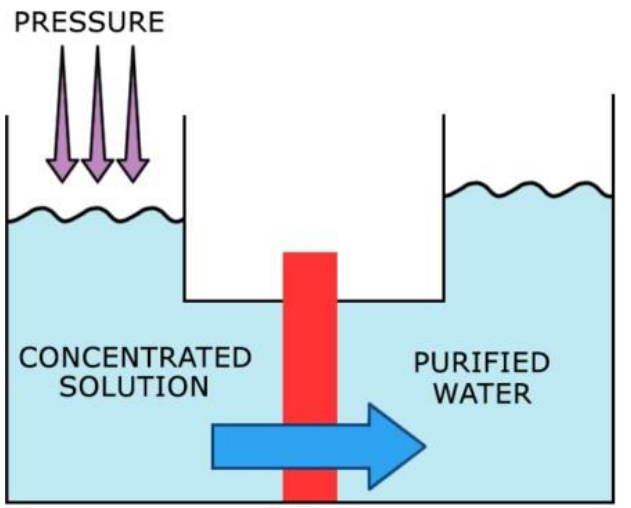

Fig 1. Schematic diagram of reverse osmosis process

In order to establish the flow of water through membrane in reverse osmosis process, the hydrostatic pressure applied to concentrated solution, which is termed as feed solution, must be greater than osmotic pressure[7]. The water or solvent flow from feed side through membrane in reverse osmosis process to permeate side is divided into three stages; absorption onto surface of membrane in feed side, diffusion through membrane thickness, and desorption from membrane surface in permeate side. The absorption of water onto surface of reverse osmosis membrane can be enhanced by having high hydrophilicity property of membrane. On the other hand, the process of solvent or water mobility through the thickness of membrane can be explained in either Brownian diffusion, flush, or jump diffusion [10] As hydrostatic pressure being greater than osmotic pressure to migrate the solvent to permeate side, reverse osmosis utilizes energy during the process. Amount of energy employed also depends on the water movement through the membrane. The more compact the membrane, the longer the distance of water in the membrane. Hence, the higher energy needed in the process for more compact membrane [11].

The reverse osmosis process has been commonly applied on water treatment[12]. The reason behind this is due to advantages that reverse osmosis offers for such application. Apparently, the efficiency of the process and its sustainability without ignoring the quality delivered and environmentally friendly are critical criteria to be considered in Chemical Engineering industry. In water treatment process such as desalination process, reverse osmosis process was reported to be the most energy efficient when it is compared to other technologies[13]. This makes reverse osmosis process to be preferred in water treatment process, especially in desalination process when energy is the most important concern. Specifically, energy is related to hydrostatic pressure that is applied in osmosis process in order to enhance the flow of solvent or water to the permeate side from feed side through semi permeable membrane. The hydrostatic pressure for seawater treatment is in the range of 2300 to $3500 \mathrm{kPa}$, while hydrostatic pressure to treat brackish water is in the range of $100-300 \mathrm{kPa}[14]$, [15] Beside advantageous energy efficiency of reverse osmosis that makes it preferred compared to other treatment, there are other advantages of using reverse osmosis. The advantages are that reverse osmosis has high water permeability, high salt rejection, which is applicable in seawater treatment, and also it satisfies the standard rules of public health, environmental concerns, and separation process[16].

\section{Literature Review}

In spite of the advantageous properties that reverse osmosis offers in its application, there are still challenges that membrane technology especially reverse osmosis (RO) membrane technology faces. During its utilisation in treating feed solution to get permeate, reverse osmosis (RO) membrane is found to be at risk of experiencing fouling. Fouling phenomenon also happens in other membrane technology beside reverse osmosis membrane technology. Fouling is deposition of unwanted mass on membrane surface or in the membrane thickness, which causes a decline in the membrane performance such as its permeation flux and salt rejection. From the definition, fouling phenomenon can be categorised according to the place where it happens. There is surface fouling, which happens on the surface of membrane, and internal fouling, which is inside the membrane[1], [8]. The effects of fouling phenomenon in membrane technology beside on flux and rejections are decline in the productivity and permeate quality that cause rise of operating cost due to additional cleaning process. Other than that, fouling also causes increase in energy demand, additional of pre-treatment, its removal process from membrane, extra maintenance necessary, and it decreases life time of membrane. The schematic diagram figuring the surface and internal fouling are shown in Figure 2[17].

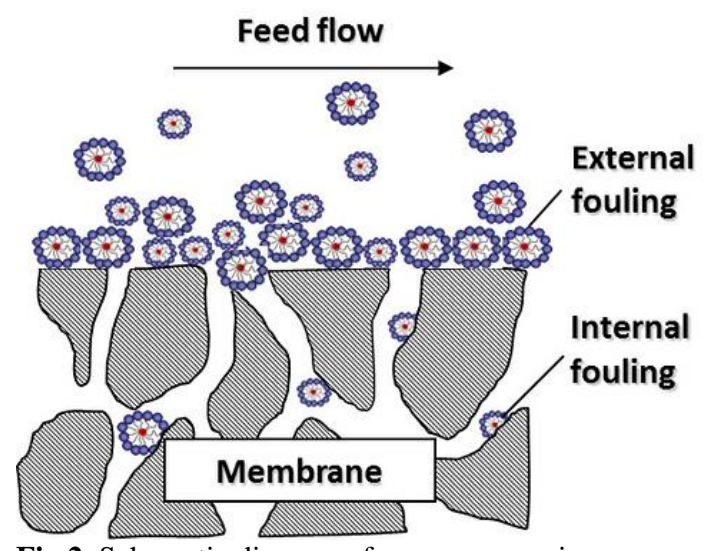

Fig 2. Schematic diagram of reverse osmosis process

Specifically, the fouling phenomenon that happens in reverse osmosis process is categorised as high-pressure membrane fouling. Moreover, when the location of fouling that takes place in or on the membrane of the RO process is taken into consideration, surface 
fouling is found to be more likely to happen because the compact relativeness and the nature of RO membrane, which is nonporous[11]. In addition to that, the surface fouling on RO membrane was reported to be more reversible compared to internal fouling in the membrane. The reversibility of fouling in reverse osmosis membrane both for internal and surface fouling are dependent on the feed solution conditions such as its components and feed's interaction with the membrane[11]. In addition to that, the treatment of feed solution differs from one to another hence the difficulty is also different. In addition to surface and internal foulings, according to the foulant types there are various types of foulings; bio-fouling, organic fouling, inorganic fouling, and colloidal fouling[13]. The mechanisms and factors that affect foulings are different from one to another.

One of the foulings that happen in RO process is biofouling. Biofouling is deposition or formation of biofilm on membrane or in the membrane to a degree of unacceptable quantity. The degree of complexity of biofouling is more than other foulings as its composition is bacteria, and extracellular polymeric substance (EPS); all together this makes biofouling to cause high operating cost when reverse osmosis is being proceeded. Three steps biofouling formation in the reverse osmosis process is bacteria attachment, reproduction, and detachment[11]. Biofouling is reported to be the most common fouling that happen in Reverse Osmosis membrane process[18].

In the first step, bacteria as the main cause of biofouling approaches the membrane and then adheres itself to the membrane[11]. According to Jiang et al., (2017), membrane characteristics; its charge, hydrophobicity, morphology, roughness, and its compositions are one of the factors. The other factors are microbial properties; charge, structure, and operating conditions that includes temperature, pressure, and $\mathrm{pH}$. The next step in biofouling process is the microorganism reproduction. In this step, the microorganism consumes nutrient that is provided in the feed solution or might be on the membrane and it undergoes proliferation while at the same time excreting extracellular polymeric substance (EPS). Beside nutrient consumption, the excreted EPS is essential in this step for biofouling as this substance makes the biofouling become stronger thus it becomes more difficult to be cleaned up. In order to sustain its existence on the surface or in the membrane, the microorganism then detaches itself from the populated biofilm of biofouling to find other vacant sites to grow another fouling site[11]. The microorganism repeats the cycle of the three steps once it finds suitable place or site on the surface or in the membrane. Concerning the last step, which is detachment step, this was reported to be the most difficult to be controlled in for the cleaning purpose. In the other first two steps, there are necessary requirements in order to achieve the purpose. Bacteria or microorganism must be in the composition of feed, the second requirement is that there must be vacant space on the surface or in the membrane, and the microorganisms needs nutrient in order to enhance their lives and for reproduction[11].

\section{Methods}

With the discovered fouling that act as challenges to Reverse Osmosis membrane, it threatens the performance of membrane performing its function in Reverse Osmosis process. On the top of that, through the membrane fouling, it may deflect or even break the structure of membrane including RO membrane. To the further extend, RO membrane may lose its function completely and has no function in Reverse Osmosis process.

In this review, the fouling of discovered Reverse Membrane is identified with the negative effects caused by the fouling to the membrane. Moreover, the types of RO membrane were also elaborated in comprehensive manner in order to identify each one of the foulings' traits and the negative effects to the membrane.

Having identified the types in Reverse Osmosis membrane fouling, the currently discovered control solutions to the challenges were also classified. The possible control solutions to the Reverse Osmosis membrane fouling are determined accordingly based on the its application on the Reverse Osmosis membrane. In this review, various Reverse Osmosis membrane fouling control were identified and classified into its application, material and other characteristics.

\section{Results and Discussion}

\subsection{RO Membrane Fouling \\ 4.1.1 Organic Fouling}

Another type of fouling in reverse osmosis membrane technology is organic fouling caused by organic material. Organic fouling is the major problem that occur in treating wastewater with reverse osmosis membrane. This is due to the fact that drainage water contains a lot more organic material than the concentration of organic material in other surface water. Organic foulant is composed of generally protein such as Bovine Serum Albumin (BSA), polysaccharides (alginate), and humic acid. Similar to biofouling, there are factors that affect formation organic fouling on the surface of reverse osmosis membrane. Main factors affecting organic fouling are feed solution chemistry; type of solute that it contains the concentrations, the foulant that is contained in the feed solution interaction with the membrane, and the interaction between one foulant to another foulant that potentially makes organic fouling more difficult to be cleaned[11]. From the factors that affect the organic fouling formation, membrane chemistry also plays important role in it, whether it is more likely to be adhered by the organic material or not. In addition to that, since the membrane used in reverse osmosis process is nonporous membrane, organic fouling only happens on the surface of the membrane rather forming in the thickness of membrane[11].

Formation of organic fouling on the surface of reverse osmosis membrane is divided into three steps. The steps of organic fouling formation are deposition of organic material onto surface, same type organic material interaction, and possibly different type foulants interaction. The last step potentially causes a great decrease of reverse osmosis membrane performance such as membrane flux when complex structure is formed between one organic materials interacted with other substance or other organic material. During its formation, relatively low molecular weight of organic material is formed in the basic stage. During this stage, the organic material, which has low molecular weight, on the surface of reverse osmosis membrane is more difficult to be cleaned[11]. Meanwhile, large part of organic fouling formed on the membrane surface was caused by high molecular weight organic material that is more than $50000 \mathrm{Da}$.

\subsubsection{Inorganic Fouling}

Inorganic fouling is a type of fouling that takes place on the surface and in the thickness or reverse osmosis membrane. Inorganic fouling or scaling is formation or deposition of inorganic substance such as salt on the membrane or inside the pore of the membrane. Inorganic fouling happens as the concentration of ion exceeds its equilibrium solubility in the fees solution, resulting saturation process. Hence, deposits on the surface or in the pore of membrane. Moreover, the process of inorganic fouling or scaling in reverse osmosis undergoes some stages, which are nucleation stage after specific ions pass equilibrium concentration, and then homogeneous or heterogeneous crystal growth process. Iron was found to be the easiest element to be deposited on RO membrane when it is compared 
with calcium and magnesium[11]. Factors that affect the formation of inorganic foulings are reported to be the feed solution composition and the concentration, low direction of feed with respect to membrane, and the membrane characteristics towards the ions in the feed solution. An example of inorganic fouling is easy to take place is the deposition of calcium phosphate on and in the reverse osmosis membrane. The deposition of the material happens on and in the membrane, thus cleaning and high maintenance cost of the membrane is necessary.

\subsubsection{Colloidal Fouling}

The fourth type of fouling that happens in reverse osmosis membrane process is colloidal fouling. This type of fouling is fundamentally caused by colloids itself or particles that develop on the membrane. There are materials that potentially cause colloidal fouling on reverse osmosis membrane such as silica, protein, and iron oxide. Colloidal fouling is extensively divided into inorganic fouling and organic macromolecules, depending on the type of colloids or particles that deposit on the membrane[11]. Aluminium silicate, silica, iron oxide or iron hydroxide in water are examples of inorganic material or foulant in colloidal fouling. On the other hand, organic materials such as polysaccharides, protein, and other organic material in water are examples of colloidal foulants on reverse osmosis membrane[3]. Factors affecting colloidal foulings are the feed solution chemistry or its characterization, membrane characterization that make fouling tend to happen on the surface of the membrane or not. The other important factors that affect the fouling are material shape, its size, the charge of the material that make it repulsive or attractive to membrane[11].

\subsection{Fouling Control Methods}

\subsubsection{Pre-Treatment}

Despite of foulings that have been identified, there has been efforts to control or even to eliminate the unwanted phenomena. Some methods have been studied and applied on fouled reverse osmosis membrane. Namely, the methods to control fouling in reverse osmosis process are pre-treatment, membrane monitoring, membrane cleaning, surface modification, and developing novel reverse osmosis membrane[19]-[23]. In pre-treatment method, the feed solution is treated so that it causes less harm to the RO membrane or even eliminates foulants. According to the operating conditions such as the feed solution and membrane chemistry, there are commonly used techniques in pre-treatment methods; photo oxidation, disinfection, coagulation, scale inhibitor, ion exchange resins, granular media, and membrane treatment.

\subsubsection{Membrane Monitoring}

Beside pre-treatment, which focuses on the feed solution, the other control methods in RO foulings focuses on the membrane. The first method is membrane monitoring method. This method monitors membrane when there are signs of fouling happens on or in the membrane. In situ monitoring is not really efficient as it usually detects the fouling when it is at advanced stage[11]. The detection method usually depends on the performance of RO membrane, which are flux and permeate flux. Another way is to stop the process and examine the membrane to check on foulings properties and compositions[24], [25]. There are some breakthroughs in this method such as ultrasonic time-domain reflectometry (UTDR), scale observation detector (EXSOD), and electrical impedance spectroscopy (EIS)[11]. UTDR is a real time in situ technique that utilizes ultrasonic wave to determine fouling layer thickness based on its reflection (Jiang et al., 2017). This technique was reported to be not applicable in detecting biofouling[11]. While EXSOD, it is an ex situ technique that uses high resolution of digital photography to differentiate fouling phenomenon; this is applicable to be used in large scale since it detects fouling before decline in RO membrane performance happens[5], [11]. On the other side, EIS employs electrode to recognise the change of electrical property of membrane as fouling takes place. However, this technique has only been tested in laboratory scale[11].

\subsubsection{Membrane Cleaning}

In pair with membrane monitoring, membrane cleaning is the sequential method. From its name, it implies that cleaning the membrane is the technique. This method can be allocated into different means; chemical, physical, biological, and enzymatic cleaning[26]. The choice of techniques in the membrane cleaning is based on the necessary actions and fouling types that need to be cleaned. Chemical based cleaning technique is useful in breaking up the foulant layer[27]. This technique for membrane cleaning is chosen based on fouling type, membrane and fouling chemical properties, possible damage that may cause to membrane, and the cost that it makes[11]. Chemicals that are commonly used are sodium hydroxide, which is for biofouling and organic foulings, $\mathrm{HCl}$, nitric acid, sulphuric acid, and ethylene diamine tetra acetic acid or EDTA[11], [28]. Some examples of chemical cleaning are Bovine serum albumin (BSA) in organic fouling was reported to be effectively cleaned through thermos responsive polymer agent material[11]; and Li et al., (2016) reported that oxalic acid is applicable to remove organic and inorganic fouling through ultrasonic-chemical cleaning. Apart from chemical cleaning, physical cleaning technique uses water to rinse the membrane, this technique removes foulant from membrane by applying fluid shear force[11], [27].

\subsubsection{Surface Modification}

The next method is surface modification method of RO membrane. The method modifies the membrane properties such as its charge, morphology, hydrophilicity, chemical groups that attach on membrane[11]. The purpose of surface modification technique is to increase membrane's refusal to foulings. Generally, smooth and more hydrophilic membrane tends to have high fouling resistance[11]. This is shown in Jiang et al. (2017, Figure 3). It can be inferred that (a) shows the best attachment of water and less space for foulant. However, there are instances when hydrophilic materials are attracted by hydrophilic membrane, which is the cause of membrane fouling[29]. Practical application of surface modification of RO membrane is surface coating that uses physical means, and surface grafting which is based on chemical change[11]. Through RO membrane surface modification, it was found out that it reduces microorganism or bacteria that attach on the membrane, which is shown in Figure 2. (Saeki et al., 2014). Apart from the advantages that it serves, there is still drawback that this method has. By modifying the surface of RO membrane, water flux is being reduced, which is negative impact of RO membrane performance[11]. 


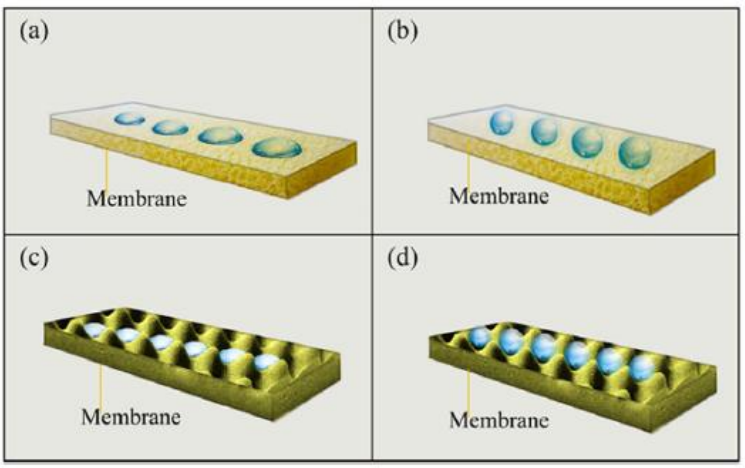

Fig 3. Smooth and hydrophilic schematic diagram on RO membrane (a) smooth and hydrophilic (b) smooth and hydrophobic (c) rough and hydrophilic (d) rough and hydrophobic

\subsubsection{Novel Membrane Material}

Addition of material to membrane or substitution of membrane material is called novel membrane material method. The most commonly used material for RO membrane is polyamide thin film composite; in this method it is replaced with materials such as Nano porous graphene, carbon nanotubes, Zwitterionic material, and metal oxide[11]. The reason behind its replacement is that polyamide is vulnerable to the existence of chlorine in feed solution[11]. Moreover, among materials that has been enhanced in this method, nanomaterial is known as the most commonly used material; due to its advantageous properties as membrane performance and antifouling[11]. Some examples of studied materials and the performances in this method are Nano-sized silver that showed resistance to fouling caused by microbial; Zwitterionic and carbon nanotubes, which are known to behave as antifouling material[11]. In addition to that, polyvinyl alcohol and Gum Arabic combination was also found to show chlorine resistance and anti-biofouling properties[11], [30]. An example of illustration of the comparison between coated and uncoated membrane is illustrated in Figure 4[11]. Nonetheless, these examples are still being studied in laboratory scale and further investigations need to be made in large scale application[11].

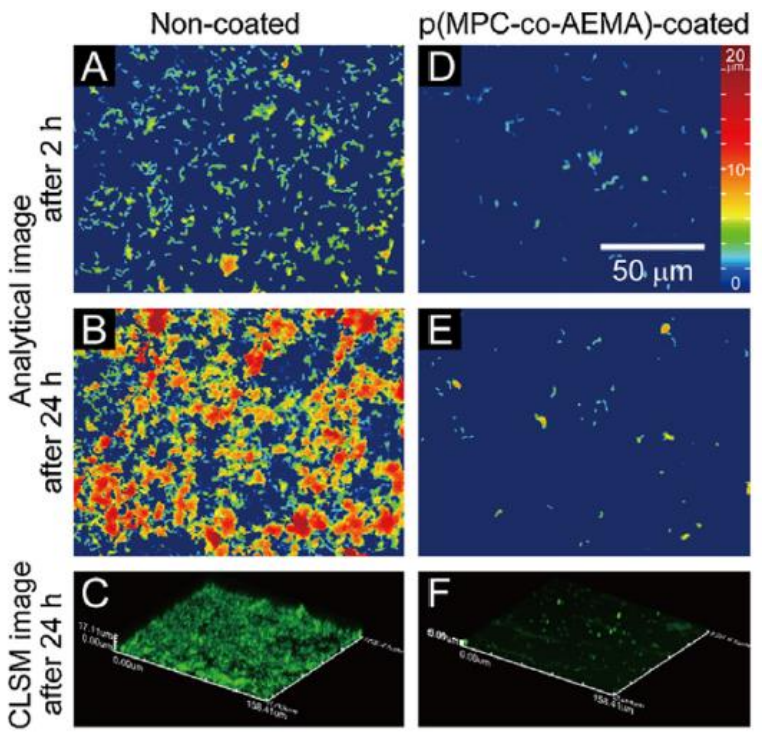

Fig 4. Comparison between coated and non-coated membrane; bacteria are shown in red, green, and yellow dots

\section{Conclusion}

To conclude, reverse osmosis (RO) membrane has been widely used as water treatment technology. Its efficiency and maintenance are some of important factors to be considered. Fouling is one of issues that defects the performance of RO membrane and its lifetime in the process. Biofouling, organic fouling, inorganic fouling, and colloidal fouling are types of fouling that happen occasionally in RO membrane process in accordance with feed chemistry, membrane type, and other conditions. In order to encounter this, several methods have been studied and developed to control or further eliminate the foulings. The methods are pre-treatment, membrane monitoring, cleaning, modification, and developing novel RO membrane. Up until now, there is method that can solely solve fouling problems; they are usually combined to get the maximum annihilation of fouling in RO membrane process. In spite of that, there are potential methods that can eliminate fouling yet they still have to be investigated thoroughly.

\section{References}

[1] L. Lin, C. Feng, R. Lopez, and O. Coronell, "Identifying facile and accurate methods to measure the thickness of the active layers of thin-film composite membranes-A comparison of seven characterization techniques," J. Memb. Sci., vol. 498, pp. 167-179, 2016.

[2] J. M. Ochando-Pulido, M. D. Victor-Ortega, and A. Martinez-Ferez, "On the cleaning procedure of a hydrophilic reverse osmosis membrane fouled by secondary-treated olive mill wastewater," Chem. Eng. J., vol. 260, pp. 142-151, 2015.

[3] F. Tang, H.-Y. Hu, L.-J. Sun, Y.-X. Sun, N. Shi, and J. C. Crittenden, "Fouling characteristics of reverse osmosis membranes at different positions of a full-scale plant for municipal wastewater reclamation,” Water Res., vol. 90, pp. 329-336, 2016. 
[4] A. R. Bartman, E. Lyster, R. Rallo, P. D. Christofides, and Y. Cohen, "Mineral scale monitoring for reverse osmosis desalination via real-time membrane surface image analysis," Desalination, vol. 273, no. 1, pp. 64-71, 2011.

[5] H. Gu, A. R. Bartman, M. Uchymiak, P. D. Christofides, and Y. Cohen, "Self-adaptive feed flow reversal operation of reverse osmosis desalination," Desalination, vol. 308, pp. 63-72, 2013.

[6] M. Yao, D. Yan, P. Kabat, H. Huang, R. W. A. Hutjes, and S. E. Werners, "Analysing monthly sectorial water use and its influence on salt intrusion induced water shortage in urbanized deltas," Sustain. Cities Soc., vol. 26, pp. 255-263, 2016.

[7] N. K. Rastogi, A. Cassano, and A. Basile, "Water treatment by reverse and forward osmosis," in Advances in Membrane Technologies for Water Treatment, Elsevier, 2015, pp. 129-154.

[8] Q. She, R. Wang, A. G. Fane, and C. Y. Tang, "Membrane fouling in osmotically driven membrane processes: A review," J. Memb. Sci., vol. 499, pp. 201-233, 2016.

[9] R. Mcllvaine, "Reverse osmosis," Chemical 2008. http://www.idreco.com/reverseosmosis/Jamal,M.,Tasneem,U.,Hussain,T.\&Andleeb,S.2015.Bacterial_biofilm_:its_composition,_fo rmationan_drole_in_human_infections._RRJMB,4,1-15 (accessed Sep. 09, 2018).

[10] W. Gao et al., "Understanding water and ion transport behaviour and permeability through poly (amide) thin film composite membrane," J. Memb. Sci., vol. 487, pp. 32-39, 2015.

[11] S. Jiang, Y. Li, and B. P. Ladewig, "A review of reverse osmosis membrane fouling and control strategies," Sci. Total Environ., vol. 595, pp. 567-583, 2017.

[12] Z. Yang, Y.-X. Sun, T. Ye, N. Shi, F. Tang, and H.-Y. Hu, "Characterization of trihalomethane, haloacetic acid, and haloacetonitrile precursors in a seawater reverse osmosis system," Sci. Total Environ., vol. 576, pp. 391-397, 2017.

[13] G.-R. Xu, J.-N. Wang, and C.-J. Li, "Strategies for improving the performance of the polyamide thin film composite (PA-TFC) reverse osmosis (RO) membranes: Surface modifications and nanoparticles incorporations," Desalination, vol. 328, pp. 83-100, 2013.

[14] A. Sagle and B. Freeman, "Fundamentals of membranes for water treatment," Futur. Desalin. Texas, vol. 2, no. 363, p. 137, 2004.

[15] R. H. Perry and D. W. Green, "Prerry's Chemical Engineers Handbook (7th Eds.)." New York: McGraw-Hill, 1997.

[16] J. A. López-Ramírez, M. D. C. Oviedo, and J. M. Q. Alonso, "Comparative studies of reverse osmosis membranes for wastewater reclamation," Desalination, vol. 191, no. 1-3, pp. 137-147, 2006.

[17] SHAMS, "Membrane Fouling Diagram," 2013. http://www.shamskm.com/blogs/?attachment_id=17208 (accessed Sep. 06, 2018).

[18] NTECL, "Desalination Plant - An Overview." http://ntpcntecljv.co.in/knowledge/desalination.html (accessed Sep. 08, 2018).

[19] R. A. Al-Juboori and T. Yusaf, "Biofouling in RO system: mechanisms, monitoring and controlling,” Desalination, vol. 302, pp. 1$23,2012$.

[20] A. Brehant, V. Bonnelye, and M. Perez, "Comparison of MF/UF pretreatment with conventional filtration prior to RO membranes for surface seawater desalination," Desalination, vol. 144, no. 1-3, pp. 353-360, 2002.

[21] L. Henthorne and B. Boysen, "State-of-the-art of reverse osmosis desalination pretreatment," Desalination, vol. 356, pp. 129-139, 2015.

[22] T. Nguyen, F. A. Roddick, and L. Fan, "Biofouling of water treatment membranes: a review of the underlying causes, monitoring techniques and control measures," Membranes (Basel)., vol. 2, no. 4, pp. 804-840, 2012.

[23] S. Robinson, S. Z. Abdullah, P. Bérubé, and P. Le-Clech, "Ageing of membranes for water treatment: Linking changes to performance," J. Memb. Sci., vol. 503, pp. 177-187, 2016.

[24] X. Xu, F. Liu, L. Jiang, J. Y. Zhu, D. Haagenson, and D. P. Wiesenborn, "Cellulose nanocrystals vs. cellulose nanofibrils: a comparative study on their microstructures and effects as polymer reinforcing agents,” ACS Appl. Mater. Interfaces, vol. 5, no. 8, pp. 2999-3009, 2013.

[25] M. A. Sari and S. Chellam, "Reverse osmosis fouling during pilot-scale municipal water reuse: Evidence for aluminum coagulant carryover," J. Memb. Sci., vol. 520, pp. 231-239, 2016.

[26] T. Koo, Y. J. Lee, and R. Sheikholeslami, "Silica fouling and cleaning of reverse osmosis membranes," Desalination, vol. 139, no. $1-3$, pp. 43-56, 2001.

[27] G. Z. Ramon, T.-V. Nguyen, and E. M. V Hoek, "Osmosis-assisted cleaning of organic-fouled seawater RO membranes," Chem. Eng. J., vol. 218, pp. 173-182, 2013.

[28] A. Al-Amoudi and R. W. Lovitt, "Fouling strategies and the cleaning system of NF membranes and factors affecting cleaning efficiency," J. Memb. Sci., vol. 303, no. 1-2, pp. 4-28, 2007.

[29] B. Kwon, S. Lee, J. Cho, H. Ahn, D. Lee, and H. S. Shin, "Biodegradability, DBP formation, and membrane fouling potential of natural organic matter: Characterization and controllability," Environ. Sci. Technol., vol. 39, no. 3, pp. 732-739, 2005.

[30] W. Falath, A. Sabir, and K. I. Jacob, "Novel reverse osmosis membranes composed of modified PVA/Gum Arabic conjugates: Biofouling mitigation and chlorine resistance enhancement," Carbohydr. Polym., vol. 155, pp. 28-39, 2017. 